\title{
Erratum: Comparison of incoherent operations and measures of coherence [Phys. Rev. A 94, 052336 (2016)]
}

\author{
Eric Chitambar and Gilad Gour \\ (Received 23 December 2016; published 12 January 2017)
}

DOI: 10.1103/PhysRevA.95.019902

Theorem 21 is not correct. The described algorithm for generating IO Kraus operators does not always succeed. Namely, when repeating the previous steps in step (5), the established condition $\left\langle 0\left|\tilde{M}_{j}\right| x\right\rangle\left\langle 1\left|\tilde{M}_{j}\right| x\right\rangle=0$ may no longer hold. We thank Xiao-Dong Yu for bringing this to our attention as well as for providing an explicit counterexample,

$$
\begin{aligned}
& M_{0}=\frac{1}{\sqrt{2}}(|0\rangle\langle 0|+|+\rangle\langle 1|), \\
& M_{1}=\frac{1}{\sqrt{2}}(|1\rangle\langle 0|+|-\rangle\langle 1|),
\end{aligned}
$$

where $| \pm\rangle=\frac{1}{\sqrt{2}}(|0\rangle \pm|1\rangle)$. These are Kraus operators for a MIO channel which is not IO.

Theorem 21 plays a crucial role in our proof for Theorem 24, a main result of the paper. Nevertheless, Theorem 24 still holds true as we will now show by a different proof.

Theorem 24. For qubit states $\rho$ and $\sigma$, the transformation $\rho \rightarrow \sigma$ is possible by either SIO, DIO, IO, or MIO if and only if both $C_{R}(\rho) \geqslant C_{R}(\sigma)$ and $C_{R, \Delta}(\rho) \geqslant C_{R, \Delta}(\sigma)$.

Proof. By Lemma 23, these conditions are sufficient for the transformation $\rho \rightarrow \sigma$ by SIO (and therefore also by DIO, IO, MIO). Conversely, as shown in Sec. II A 2, $C_{R}$ is a MIO monotone. What remains to be shown is that $C_{R, \Delta}$ is also a MIO monotone.

To prove monotonicity of $C_{R, \Delta}$ for general transformations, we only need to consider special transformations of the form

$$
\rho=\frac{1}{2}\left(\begin{array}{ll}
1 & r \\
r & 1
\end{array}\right) \rightarrow \sigma=\left(\begin{array}{cc}
q & t \\
t & 1-q
\end{array}\right)
$$

Indeed, suppose that $\rho^{\prime}=\left(\begin{array}{ll}p & s \\ s & 1-p\end{array}\right)$ is an arbitrary standard-form qubit state that can be converted into $\sigma$ by MIO. Then the state $\rho=\frac{1}{2}\left(\begin{array}{cc}1 & s / \sqrt{p(1-p)}\end{array}\right)$ satisfies $C_{R}(\rho) \geqslant C_{R}\left(\rho^{\prime}\right)$ and $C_{R, \Delta}(\rho)=C_{R, \Delta}\left(\rho^{\prime}\right)$. Hence the transformation $\rho \rightarrow \rho^{\prime}$ is possible by MIO, and therefore also the transformation $\rho \rightarrow \sigma$. Since $\rho$ and $\rho^{\prime}$ have the same values of $C_{R, \Delta}$, we see that if $C_{R, \Delta}$ is a monotone under a MIO when the initial state has uniform diagonal elements, then it must also be a monotone for general initial states.

To prove monotonicity for transformation (2), we study the Choi matrix $\Omega^{A B}=\mathcal{E}^{A} \otimes \mathbb{I}^{B}\left(|\Phi\rangle\left\langle\left.\Phi\right|^{A B}\right)\right.$ for a general MIO map $\mathcal{E}$, where $|\Phi\rangle=|00\rangle+|11\rangle$. The MIO conditions on $\mathcal{E}$ translate into the following conditions on $\Omega^{A B}$ :

$$
\begin{aligned}
\operatorname{Tr}_{B}\left[\mathbb{I} \otimes|y\rangle\langle y| \Omega^{A B}\right]-\Delta\left(\operatorname{Tr}_{B}\left[\mathbb{I} \otimes|y\rangle\langle y| \Omega^{A B}\right]\right) & =0, \quad y=0,1, \\
\operatorname{Tr}_{A} \Omega^{A B} & =\mathbb{I}, \\
\Omega^{A B} & \geqslant 0 .
\end{aligned}
$$

Represent $\Omega^{A B}$ in the Pauli basis as

$$
\Omega^{A B}=\frac{1}{2}\left(\mathbb{I} \otimes \mathbb{I}+\vec{a} \cdot \vec{\sigma} \otimes \mathbb{I}+\mathbb{I} \otimes \vec{b} \cdot \vec{\sigma}+\sum_{i j} c_{i j} \sigma_{i} \otimes \sigma_{j}\right) .
$$

Then conditions (3) and (4) require that

$$
\begin{aligned}
\vec{b} & =0, \\
a_{x} & =a_{y}=c_{x z}=c_{y z}=0 .
\end{aligned}
$$

Since we consider transformation of real density matrices, we can assume without loss of generality that $\Omega^{A B}$ is real [i.e., that $\left.\left(\Omega^{A B}\right)^{T}=\Omega^{A B}\right]$. Thus,

$$
c_{y x}=c_{x y}=c_{z y}=0
$$


Additionally, since the initial state in transformation (2) is invariant under conjugation by $\sigma_{x}$, we can also assume that $\Omega^{A B}$ is invariant under conjugation by $\mathbb{I} \otimes \sigma_{x}$. This further yields the condition

$$
c_{y y}=0 .
$$

The remaining free parameters are $\left\{a_{z}, c_{x x}, c_{z x}, c_{z z}\right\}$, and the smallest eigenvalue of $\Omega^{A B}$ can be explicitly computed as

$$
\begin{aligned}
0 \leqslant \lambda_{\min }\left(\Omega^{A B}\right) & =\frac{1}{2}\left(1-\sqrt{\left.c_{x x}^{2}+c_{z x}^{2}+c_{z z}^{2}+a_{z}^{2}+2 \sqrt{c_{x x}^{2} c_{z z}^{2}+a_{z}^{2}\left(c_{z x}^{2}+c_{z z}^{2}\right)}\right)}\right. \\
& \leqslant \frac{1}{2}\left(1-\sqrt{c_{x x}^{2}+c_{z x}^{2}+a_{z}^{2}+2\left|a_{z} c_{z x}\right|}\right)
\end{aligned}
$$

where the second inequality is obtained by setting $c_{z z}=0$ in the first line. Therefore, $\Omega^{A B} \geqslant 0$ requires that

$$
\begin{aligned}
c_{x x}^{2} & \leqslant 1-\left(a_{z}^{2}+c_{z x}^{2}+2\left|a_{z} c_{z x}\right|\right) \\
& \leqslant 1-\left(a_{z}^{2}+c_{z x}^{2} r^{2}+2\left|a_{z} c_{z x}\right| r\right) \\
& \leqslant 1-\left(a_{z}+r c_{z x}\right)^{2},
\end{aligned}
$$

for $0 \leqslant r \leqslant 1$.

Now, since $\rho=\frac{1}{2}\left(\mathbb{I}+r \sigma_{x}\right)$, we have

$$
\sigma=\operatorname{Tr}_{B}\left[\mathbb{I} \otimes \rho \Omega^{A B}\right]=\frac{1}{2}\left[\mathbb{I}+\left(a_{z}+r c_{z x}\right) \sigma_{z}+r c_{x x} \sigma_{x}\right] .
$$

The $\Delta$ robustness of $\sigma$ therefore satisfies

$$
C_{R, \Delta}(\sigma)=\frac{r c_{x x}}{\sqrt{\left[1+\left(a_{z}+r c_{z x}\right)\right]\left[1-\left(a_{z}+r c_{z x}\right)\right]}}=r \frac{c_{x x}}{\sqrt{1-\left(a_{z}+r c_{z x}\right)^{2}}} \leqslant r=C_{R, \Delta}(\rho),
$$

where the last inequality follows from Eq. (12). This completes the proof.

Notice that $C_{R, \Delta}$ remains invariant under MIO if and only if $c_{z z}=c_{z x}=0$ and $c_{x x}=\sqrt{1-a_{z}^{2}}$. For example, for the transformation $\rho=\frac{1}{2}\left(\begin{array}{cc}1 & s / \sqrt{p(1-p)} \\ s(1-p) & 1\end{array}\right) \rightarrow \rho^{\prime}=\left(\begin{array}{ll}p & s \\ s & p\end{array}\right)$ described above, one takes $a_{z}=2 p-1, c_{x x}=2 \sqrt{p(1-p)}$, and $c_{z z}=c_{z x}=0$. 